\title{
Materials Science and Engineering Study Panelists
}

The following is a list of the chairmen, vice-chairmen, and pa nelists for the national Materials Science and Engineering Study commissioned by the National Research Council at the request of the National Academy of Sciences and the National Academy of Engineering.

\section{Panel 1-Materials Research Opportunities and Needs in MSE}

James S. Langer, University of California, Santa Barbara, Chairman Ceorge Parshall, E.I. du Pont de Nemours \& Co., Inc., Vice Chairman James O. Stiegler, Oak Ridge National Laboratory, Vice Chairman

Sumner A. Barenberg, E.l. du Pont de Nemours \& Co., Inc

Elias Burstein, University of Pennsylvania

Peter Cannon, Rockwell International Mildred Dresselhaus, Massachusetts Institute of Technology

James Economy, IBM Corporation
George S. Hammond, Allied-Signal Inc.

Arthur H. Heuer, Case Western Reserve University

John P. Hirth, Ohio State University

Pierre C. Hohenberg, AT\& T Bell

Laboratories

Ian Hughes, Inland Steel Co. Research Laboratory

Robert 1. Jaffee, Electric Power Research Institute

John D. Joannopoulos, Massachusetts Institute of Technology
Herbert H. Johnson, Cornell University

Robert A. Laudise, AT\&T Bell

Laboratories

Christopher Magee, Ford Motor Company

E. Ward Plummer, University of Pennsylvania

Robert Stratton, Texas Instruments James R. Rice, Harvard University

Gareth Thomas, University of California Mark Wrighton, Massachusetts Institute of Technology

\section{Panel 2-Exploitation of MSE and Technology for National Welfare}

Alan G. Chynoweth, Bell

Communications Research, Inc. Chairman

R. Glen Kepler, Sandia National Laboratories, Vice Chairman

James C. Williams, Carnegie-Mellon

University, Vice Chairman

Joseph D. Andrade, University of Utah

Mylle H. Bell, Bell South Corporation

Joel Clarke, Massachusetts Institute of

Technology

L. Eric Cross, Pennsylvania State University

Theodore Geballe, Stanford University

Gordon H. Geiger, North Star Steel

Frank E. Jamerson, General Motors

Research Laboratories

Harry A. Lipsitt, AFWAL, Wright

Patterson Air Force Base

James L. McCall, Battelle Columbus

Division

\section{Panel 3-International Cooperation and Competition in MSE}

Lyle Schwartz, National Bureau of Standards, Chairman

W. Dale Compton, National Academy

of Engineering, Vici Chairman

Rustum Roy, Pennsylvania State

University, Vice Chairman

Jordan Baruch, Jordan Baruch

Associates

Peter Bridenbaugh, Aluminum

Company of America

\section{Panel 4-Research Resources in MSE}

Terry L. Loucks, Norton Company, Chnirman

Martin Blume, Brookhaven National

Laboratory, Vice Chairman

Ceorge Whitesides, Harvard University, Vice Chairman

Bill R. Appleton, Oak Ridge National

Laboratory

Robert S. Bauer, Xerox Palo Alto

Research Center

H. Kent Bowen, Massachusetts Institute of Technology

Panel 5-Education in MSE

1. Melvin Bernstein, Carnegie-Mellon University, Chriman

Robert S. Hansen, Ames Laboratory, Vice Chairman

John Hulm, Westinghouse Electric Corporation, Vice Chnirma"

Diran Apelian, Drexel University

Ali S. Argon, Massachusetts Institute of Technology

Malcolm R. Beasley, Stanford University
C. Peter Flynn, University of Illinois

Richard J. Fruehan, Carnegie-Mellon University

Herbert I. Fusfeld, New York

University

Serge Gratch, GMI Institute

Rudolph Pariser, E.l. du Pont de

Nemours \& Co., Inc.

R. Byron Pipes, University of Delaware

Peter M. Eisenberger, Exxon Research and Engineering $\mathrm{Co}_{0}$.

Nicholas F. Fiore, Cabot Corporation

John Gilman, Lawrence Berkeley

Laboratory

Karl Hess, University of Illinois

Israel S. Jacobs, General Electric

Research and Development Center

J. David Litster, Massachusetts Institute of Technology

Gilbert Y. Chin, AT\& T Bell

Laboratories

Robert Clagett, University of Rhode Island

Anthony G. Evans, University of California, Santa Barbara

Leroy Eyring, Arizona State University

Hellmut Fritzsche, University of

Chicago

Bruce Harmon, Iowa State University
Thomas C. McGill, Jr., California Institute of Technology

John P. Riggs, Celanese Research Corporation

Gerd M. Rosenblatt, Lawrence Berkeley Laboratory

Palle Smidt, Microelectronics

Corporation

Robert Street, Xerox Palo Alto Research Center

Hilliard Williams, Monsanto Company

Maxine Savitz, The Garett Corporation Gabor A. Somorjai, University of California, Berkeley

Gregory Stillman, University of Illinois James J. Tietjen, RCA Laboratories Robert White, Control Data

Noel MacDonald, Cornell University Dennis McWhan, AT\& T Bell

Laboratories

Emil Pfender, University of Minnesota

Bhakta B. Rath, Naval Research

Laboratories

John S. Rydz, Emhart Corporation

Isaac F. Silvera, Harvard University

Richard Stein, University of

Massachusetts

Julia Weertman, Northwestern University

Edward J. Kramer, Cornell University Gerald L. Liedl, Purdue University

Kathleen Taylor, General Motors Research Laboratories

Edwin L. Thomas, University of Massachusetts

Richard E. Tressler, Pennsylvania State University

Kenneth G. Wilson, Cornell University 